\title{
Futuros docentes peruanos frente a la educación mediática: entre la deuda y la esperanza
}

\section{Future Peruvian teachers and media education: between debt and hope}

DOI: https://doi.org/10.32870/dse.v0i24.900

\author{
Julio-César Mateus* \\ María-Teresa Quiroz-Velasco**
}

\begin{abstract}
Resumen
Este artículo explora los conocimientos y actitudes de docentes en formación peruanos sobre la educación mediática como estrategia para formar ciudadanos mediáticos, es decir, críticos y creativos frente a las TIC para ejercer su ciudadanía. En un contexto de pandemia global en que dependemos de las tecnologías para garantizar el ejercicio del derecho a la educación y en un escenario donde los flujos de información se multiplican, resulta pertinente discutir los términos en que el estudio de las TIC debe ser incorporado en la formación inicial docente. Para conocer la voz de los futuros docentes realizamos cuatro grupos focales con algunas de las instituciones educativas más importantes del Perú. Los resultados dejan ver que los docentes son críticos del papel educativo de los medios de comunicación, pero también lo son de sus propias carencias como profesionales para comprenderlos, manejarlos, apropiarse de ellos e integrarlos en el aula, más allá de su presencia como auxiliares didácticos. En ese sentido, acusan una falta de formación sobre las TIC desde una dimensión sociocultural que supere la mirada instrumental, y sugieren la necesidad de incorporarlas en los planes de estudio, como parte de su formación integral.
\end{abstract}

Palabras claves: Educación mediática - competencia mediática - literacidad mediática - docentes - formación inicial docente - Perú.

\begin{abstract}
This article explores the knowledge and attitudes of Peruvian teachers in initial training regarding media education as a strategy to train competent citizens competent in the use of ICTs. In a context of a global pandemic where we depend on technologies to guarantee the right to education and where flows of information multiply, it is pertinent to discuss the terms on which these ICTs should be incorporated into initial teacher training. In order to know the voice of future teachers, we conducted four focus groups with some of the most important educational institutions in Peru. The results show that teachers are critical of
\end{abstract}

* Doctor en Comunicación. Líneas de investigación: educación mediática, teorías de la comunicación, culturas digitales. Universidad de Lima. Perú.jimateus@ulima.edu.pe

** Doctora en Sociología. Líneas de investigación: educación mediática, teorías de la comunicación, culturas digitales. Universidad de Lima. Perú.tquiroz@ulima.edu.pe 
the educational role of media, but also that they are aware of their own shortcomings as professionals to understand, manage, appropriate and integrate them into the classroom, beyond their presence as teaching aids. In that sense, they have little training on ICTs from a sociocultural dimension that goes beyond the instrumental view, and detect the need to incorporate ICTs in their curricula as part of their comprehensive training.

Key words: Media education - media competence - media literacy - teachers - initial teacher training Peru.

\section{Introducción}

Como en buena parte de Latinoamérica, la pandemia causada por el Covid-19 desnudó en el Perú una serie de precariedades y problemas estructurales vinculados con la brecha digital.

Se trata de un momento histórico y complejo de transformación digital, que parte de un sistema educativo con grandes brechas de calidad e infraestructura, así como un bajo rendimiento en los rankings internacionales. El acceso a Tecnologías de Información y Comunicación (TIC) parecía al alza. Según cifras oficiales, en 2019 casi todos los peruanos tenían al menos un dispositivo TIC en casa, mientras que $58.8 \%$ de la población mayor de seis años accedía a internet, lo que representa un crecimiento sostenido, pero insuficiente, en los últimos años (INEI, 2019). Con la súbita aparición de la pandemia global y la cuarentena decretada por el gobierno, el equipamiento con que contaban los hogares peruanos no alcanzó para garantizar la continuidad del derecho educativo.

Aun así, el Ministerio de Educación preparó en corto tiempo "Aprendo en Casa", una estrategia multicanal de emergencia que permite emitir contenidos educativos por TV, radio e internet. Un estudio de mayo de 2020 alertó que para $72 \%$ de la población, las condiciones de conectividad, equipamiento y preparación no eran óptimas, situación que se agravó en las zonas rurales y en los niveles socioecónicos más pobres, donde la valoración de la estrategia fue regular. Como respuesta, el Ministerio de Educación inició la compra y distribución masiva de un millón de tabletas con recarga solar y conexión a internet para llegar a la totalidad de usuarios del servicio escolar, priorizando estudiantes y docentes de zonas rurales y urbanas focalizadas.

A pesar de las brechas, la masificación de tecnologías digitales y su creciente penetración en la sociedad generan que un número mayor de personas interactúen, consuman y produzcan contenidos mediáticos. Por otra parte, una de las consecuencias más visibles de la pandemia ha sido el grado intenso con el que interactuamos con recursos tecnológicos cambiantes. Así, "la dinámica de cambio hace más relevante la importancia de la educación y de las competencias para desenvolverse en una sociedad en constante mutación" (Díaz, 2019: 3). Por eso, la escuela debe aprovechar la coyuntura de crisis para responder al propósito de preparar a "que las personas enfrenten un mundo complejo, incierto, cada vez más globalizado y desarrollado digitalmente" (Díaz, 2019: 3). 


\section{TIC y formación inicial docente}

En 2018 entró en vigor un nuevo Currículo Nacional en el Perú, que plantea una competencia específica relacionada con las TIC. Aunque la competencia mediática e informacional no es mencionada de forma explícita en el plan de estudios, muchas de sus propuestas conceptuales están presentes. Esta nueva competencia es definida como transversal, es decir, con presencia en todas las áreas curriculares, persigue la finalidad de desarrollar capacidades en los estudiantes para participar de los entornos digitales generados por las TIC. El objetivo es que los estudiantes adquieran cuatro habilidades: (i) personalizar entornos virtuales, (ii) gestionar información del entorno virtual, (iii) interactuar en entornos virtuales y (iv) crear objetos virtuales en diferentes formatos (Mateus, Suárez-Guerrero, 2017).

Para que esta "Competencia TIC" que plantea el nuevo currículo sea comprendida desde un enfoque sociocultural, más que como una habilidad instrumental (Mateus, Quiroz, 2021), es imprescindible volver a los aportes de la educación mediática (EM). Como concluyó un grupo de expertos en la Declaración de Juiz De Fora — que actualiza declaraciones anteriores como la de Grünwald, 1982; Tolouse, 1990; Viena, 1999; Alejandría, 2005; París-2007 y Moscú, 2012-, la EM resulta un asunto urgente porque su ausencia

puede ser factor de segregación y exclusión social, sea por la falta de acceso a los conocimientos producidos históricamente, por la discriminación de culturas tradicionales, por la manipulación de informaciones o por la negación de la existencia de grupos sociales desfavorecidos como sujetos históricos y de derechos (Alfamed, 2017).

A estas alturas, es claro decir que la EM ha pasado de ser una práctica pedagógica a un proyecto político relacionado con derechos humanos fundamentales como la libertad de expresión y la participación (Frau-Meigs, Velez, Flores-Michel, 2017). Para Livingstone, Wijnen, Papaioannou, Costa y Grandío (2013), el "analfabetismo mediático" está pronto a convertirse en un problema tan grave como el "analfabetismo letrado". El auge de las fake-news, sobre todo en una situación límite por causa del nuevo coronavirus, ha puesto de manifiesto la dificultad de las personas para reconocer y gestionar información, poniendo en riesgo el ejercicio de su ciudadanía e incluso de su salud. En contextos como los que atravesamos, esto resulta altamente preocupante y deben llamarnos a reflexionar sobre el sentido de educar sobre los medios, que es diferente a educar con medios (que alude más a la tecnología educativa que al desarrollo de capacidades críticas para interactuar con esta tecnología).

Ahora bien, el desarrollo de la educación en medios considera como punto clave la formación inicial docente, para que los medios no sean vistos únicamente como instrumentos didácticos para animar la transmisión de conocimientos (Andrada, 2015), sino como objetos de estudio en sí mismos, sobre los cuales debe generarse una reflexión pertinente (Aguaded, Jaramillo-Dent, 
Delgado-Ponce, 2021). Más aún cuando la agitada evolución del ecosistema mediático tecnológico aumenta la vulnerabilidad del docente ante sus propias capacidades, ocasionando que prefiera utilizar sólo aquellas herramientas que domina, sin generar un diálogo oportuno con las prácticas mediáticas de sus estudiantes fuera del entorno escolar (Suárez-Guerrero, Ros-Garrido, Lizandra, 2021). De este modo, es pertinente indagar en las percepciones docentes sobre las TIC, en tanto sus creencias modelan buena parte de sus prácticas (Tondeur y otros, 2017).

Al respecto, la propia UNESCO (2011) desarrolló un Currículo de Educación y Alfabetización de la Información para Docentes, con la mirada puesta en el desarrollo de una ciudadanía plena e informada. Esta organización, pionera en la promoción de la EM en el mundo, sumó a este currículo un nuevo marco competencial que actualiza los retos planteados por las TIC, insistiendo que las capacidades docentes del futuro incluirán idear maneras innovadoras de usar la tecnología, lo que demanda una comprensión profunda de sus posibilidades e impactos en la sociedad (UNESCO, 2019). Asimismo, en 2021 publicó una versión actualizada del currículo que motiva a organizar la formación docente en el marco de lo que denomina Alfabetización Mediática Informacional (AMI).

No obstante, persiste el reto de aterrizar estos nuevos marcos de acción en planes de estudio específicos en cada país, así como estándares internacionales validados que sirvan de orientación. El desinterés de muchos estados ha generado que la capacitación docente en EM se limite al interés de educadores y a iniciativas de proyectos aislados, antes que al desarrollo de políticas públicas (Bulger, Davison, 2018). En Europa, donde se exhiben planes más orgánicos para luchar contra las noticias falsas y para desarrollar capacidades críticas, los esfuerzos y los recursos se han centrado más en la competencia digital que en la alfabetización mediática, por lo que existe una proliferación innecesaria de diferentes marcos de alfabetización mediática que terminan por confundirnos (Buckingham, 2018). Desde esa mirada, es importante evitar caer en el reduccionismo y el sesgo tecnológico que sugiere la conceptualización exclusivamente digital y, en línea de lo propuesto por Gutiérrez-Martín y Torrego (2018), recuperar, en las nociones de alfabetización mediática y de la competencia digital, los enfoques más críticos e ideológicos característicos de la educación para los medios.

En el Perú, la formación inicial de docentes es ofrecida por tres tipos de instituciones: universidades, institutos pedagógicos y escuelas de formación artística. Actualmente, hay casi medio millón de maestros en servicio y 50 mil estudiantes de educación. 63\% de los docentes peruanos han sido formados en institutos y $36 \%$ en universidades. Hasta hoy, no es posible ofrecer un panorama homogéneo de la formación docente en cuanto a competencias educomunicativas, ya que existen muchas diferencias entre las universidades públicas frente a las privadas y las de la capital frente a las provincianas. Los problemas de conectividad, escasez de recursos financieros, infraestructura insuficiente, deficiencias administrativas, entre otras, inciden en la calidad de la formación docente y hacen difícil proponer un diagnóstico claro en esta materia (Cano, Nájar, 2019). 
En general, como concluye un trabajo reciente, el conocimiento de los futuros docentes sobre la educación mediática es muy limitado. Aquellos estudiantes de educación que no consideran haber recibido ningún tipo de formación al respecto no superan $20 \%$ del total. Apenas 16\% de 501 participantes en una encuesta aplicada en 2017, no reconocieron ningún contenido de EM en sus planes de estudio y más de la mitad (56\%) declaró no haber recibido ningún tipo de formación en este campo como parte de su entrenamiento docente (Mateus, Hernández-Breña, Figueras-Maz, 2019). Es probable que la pandemia haya causado retos aún mayores que conviene atender desde la propia formación docente.

\section{Método}

\section{Objetivo y preguntas de investigación}

El objetivo de este trabajo es explorar la dimensión subjetiva de los docentes en formación, para revelar sus ideas, expectativas y necesidades sobre la educación mediática (EM). Las preguntas de investigación sobre las que trabajamos fueron organizadas en torno a sus conocimientos sobre el tema, la formación recibida, sus actitudes frente a los medios y la posibilidad de integrar algún tipo de formación sobre ellos en las instituciones educativas en las que se forman. Esto, con la finalidad de repensar los planes de formación inicial docente para que integren de forma decida la Alfabetización Mediática e Informacional (AMI) como una materia urgente.

\section{Material}

Para responder a las preguntas, diseñamos una guía de grupo focal. Este instrumento de recolección de datos utilizó como insumo dos cuestionarios previamente validados y aplicados en las mismas instituciones, cuyos resultados han sido publicados (Mateus, Hernández-Breña, Figueras-Maz, 2019; Mateus, Hernández-Breña, 2019). Una de las ventajas de esta técnica es que permite, por un lado, contrastar ideas a partir de la interacción de diversas opiniones y generar luego un relato colectivo entre los participantes.

\section{Muestra, aplicación y codificación}

En el Perú existen 58 universidades que cuentan con grados en educación, según información publicada por la Superintendencia Nacional de Educación Superior Universitaria (SUNEDU). También, hay 356 institutos pedagógicos autorizados. A pesar del mayor número de institutos, las universidades albergan un número superior de estudiantes: alrededor de 40,434 (63.5\% del total), mientras que los institutos superiores pedagógicos albergan 23,321 estudiantes (36.5\%) (SUNEDU, 2017). En ese sentido, se buscó que los grupos focales respondieran a esta proporción.

Los criterios de selección fueron los mismos utilizados en los citados estudios, pues formaron parte del mismo proyecto de investigación (Mateus, Hernández-Breña, Figueras-Maz, 2019; Mateus, Hernández-Breña, 2019). La participación fue voluntaria y autoseleccionada, en 
respuesta a una invitación pública realizada en sus instituciones. Participaron un mínimo de 5 y un máximo de 11 estudiantes por cada institución. Las sesiones fueron grabadas en audio luego de su consentimiento informado.

Las grabaciones de los grupos focales fueron transcritos y luego codificados utilizando la herramienta NVivo. Las respuestas se codificaron buscando diferenciar a cada institución participante (a la que se asignó el rótulo "IN y el número 1, 2, 3 o 4") y el género (H para hombre o M para mujer), seguido del número del participante.

\section{Resultados}

Organizamos los resultados de los grupos focales en torno a tres ejes: (i) la concepción sobre los medios y el rol que consideran que deben asumir como docentes frente a ellos en el aula, (ii) sus percepciones sobre la competencia mediática y el reconocimiento de sus propias competencias o necesidades, así como (iii) sus ideas acerca de la integración de la educación mediática como parte de su preparación profesional inicial.

\section{Sobre el papel educativo de los medios de comunicación}

En relación con el papel que cumplen los medios, las respuestas son ambivalentes. Si bien expresan una crítica generalizada a la pobre calidad de los programas televisivos, calificándolos de vulgares, "basura" o que producen desinformación, también tienen una mirada esperanzadora sobre su potencial: "los medios, si se trabajan de una manera óptima puede transmitir valores positivos, educar, y yo estoy de acuerdo [en] que medios de comunicación se pueden hacer grandes cosas con educación" (IN3-M3).

Insisten en la idea de los medios como proveedores de información:"a través de los medios de comunicación también aprendemos y adquirimos información. Ya sea información que te va a ser útil en la vida cotidiana o no" (IN1-M2). "Porque a través [de los medios] nosotros aprendemos, nos informamos, y accedemos a internet para buscar información" (IN3-M4).

Una estudiante matizó esta apreciación, diferenciando la obtención de datos del aprendizaje:

Creo que hay una gran diferencia entre adquirir información y construir un aprendizaje, porque al momento en el que una persona adquiere información tiene datos, pero si la persona hace un proceso cognitivo, analizar esos datos de acuerdo con los conocimientos previos que yo tengo, o criticar la información es lo que va a permitir que la persona aprenda (IN1-M2).

Se constata una reiterada visión de los medios como artefactos o instrumentos: "Cuando decimos TIC pensamos en la máquina" (IN4-M3). De lo cual se desprende la exigencia de ser capacitados en habilidades para manipular softwares según su especialidad, como matemática, idiomas, arte, entre otras. 
Desde una orilla más crítica al sistema mediático, se discutió la idea del poder político o económico que está detrás de los medios.

Pierre Bourdieu menciona que el que controla los medios de comunicación tiene el poder sobre las masas. Se refería a que las personas creen mucho lo que ven a través de una pantalla, en este caso, la televisión. La tendencia es que las personas acepten como verídicas las imágenes, quedando relegada la investigación y búsqueda de fuentes, haciendo suya esa realidad y actuando de acuerdo a ello (IN1-M4).

Sin embargo, los estudiantes también evaluaron positivamente a los medios porque estimulan la creatividad: "Utilizando los medios de comunicación los estudiantes despiertan el lado imaginativo porque ahí puedes poner una realidad que no está en tu mundo real" (IN2-M3). El entronque de los medios como entorno con los contenidos curriculares podría ayudar a producir "aprendizaje significativo, porque es ahí donde el estudiante aprende verdaderamente, no solamente con el profesor y la pizarra, entonces a través de los medios de comunicación es donde el estudiante capta el propósito de una clase" (IN4-M1).

En ese contexto, otra estudiante manifestó que los medios permitían un acceso más universal al conocimiento $y$, desde luego, permitían el ejercicio de un derecho:

Creo que viene más de la necesidad de adaptarse a este mundo globalizado. Por ejemplo, estaba leyendo un artículo que decía que la Unesco plantea como un derecho universal la alfabetización universal. Entonces, creo que sí se toma esa medida si existe una gran necesidad de que las personas podamos alfabetizamos digitalmente para que le hagamos frente a este mundo globalizado (I1-M6).

\section{El medio como herramienta, el docente como mediador}

Los futuros docentes definen de forma intuitiva a los medios, básicamente como instrumentos, sin alcanzar una mirada sociocultural. Si bien la mayoría confirmó no haber oído nunca sobre la educación mediática, la asociaron al uso de los medios como recursos que les permitirían enseñar mejor. Según dijeron: "viene a ser la educación a través de los medios de comunicación" (IN4H1). De este modo, "aprovechando su propia praxis [y la] facilidad [que tienen los estudiantes hoy] de utilizar los medios de comunicación [nace la EM] para hacer más significativo el aprendizaje en ellos" (IN4-H1). Igualmente, otra estudiante la definió como "el proceso de enseñanza y aprendizaje relacionado al uso de recursos tecnológicos y medios de comunicación" (IN1-M2). Una estudiante dijo: "Imaginé algo como herramientas que servirían a los profesores para que puedan enseñar a sus alumnos" (IN2-H1), mientras que, otra estudiante compañera aseguró que la EM tenía que "ver [con] qué herramientas tecnológicas, como las aplicaciones, podemos usar nosotros [como docentes] para hacer una clase más entretenida, más didáctica" (IN2-M2).

Algunos pocos tenían otras referencias. Una estudiante que había leído a McLuhan en una asignatura de Filosofía señaló que consideraría la EM como "una educación que sale de las au- 
las, que ya no va a ser sólo el docente que va a dar la información, sino que puedes aprender por medio de la radio, televisión y diversos medios" (IN1-M4). Otra alumna definió la EM como "la preparación o formación que nos puedan brindar para poder interactuar con las nuevas tecnologías y poder hacerle frente a la sociedad de la información y conocimiento" (IN1-M7), introduciendo una mirada más relacional entre las personas y los medios. Finalmente, un par de intervenciones se refirieron puntualmente a la EM como una cuestión ligada más con la influencia de los medios: "Yo me imaginé, más o menos, en relación a la televisión con la educación, cómo se influían. Y en cuanto a las tecnologías en general, la radio, la tele también, cómo están influyendo, en qué ayudan y en qué no" (IN2-M2).

Los futuros docentes manifiestan tener presuntas capacidades innatas para interactuar con los medios, que contribuirán en su futuro ejercicio docente:

Actualmente el mundo ha revolucionado y todos los chicos desde que nacen tienen esas nociones de la tecnología inherentes a ellos mismos porque es algo que se da casi de forma automática... Por ejemplo, mi hermana tiene 8 años y ella usa WhatsApp, o sea, me escribe, me manda mensajes, yo a su edad no sabía qué era un WhatsApp, un Facebook, un Twitter, por eso ellos son más nativos a eso. Es de forma innata, nadie les enseñó (IN4-H1).

Yo no estaba muy capacitada para enseñar ese software [un software libre de edición de imágenes digitales Ilamado GIMP] porque nuestra especialidad no es Informática -estamos aprendiendo como a la fuerza, por así decirlo-, capacitándonos solos, viendo videos en YouTube, pero los niños fueron más rápidos hasta donde yo llegué, interactuaron más, prepararon presentaciones más detalladas de las que yo les hice a ellos (IN4-M3).

Una futura profesora añade que tendrán que lidiar con una permanente condición de desventaja por la velocidad irreparable de las tecnologías: "Ahora, en cada casa ya tienen una laptop, un celular, una tablet, entonces, los chiquitos son más nativos a eso, o sea, nacen, les dan un celular, y ya pueden manejarlo, pueden escribir, pueden mensajear" (IN4-M2).

Si bien los futuros docentes acusan una falta de capacitación en materia tecnológica que los pone en desventaja, también son conscientes de que su rol no será el de competir con las habilidades de sus alumnos. "Es muy importante el docente, o sea, pueden pasar muchas cosas que faciliten la vida del ser humano, pero sí o sí siempre necesitamos a un ser como guiador, como orientador" (IN4-M3). Añaden:

Siempre tiene que haber alguien que lo oriente, porque, [así] como puede ser un buen recurso, también se pueden desviar, puede no usarlo para algo bueno sino para algo malo, y ahí es donde entra la EM. Para que podamos, como docentes, capacitarlos, ya no tanto enseñarles, sino orientar para que los sepan manejar (IN4-M2). 
Les preocupa el rol del docente y precisan su papel mediador: "si el docente no enseña a moldear estos medios, entonces el alumno no seguirá el camino adecuado para adecuarlo a su contexto" (IN2-M5) y es así como la EM es concebida intuitivamente como una guía necesaria: "Mediar la interacción del estudiante con los medios que se van a trabajar. [...] el docente va a ser el que guíe los pasos que debe seguir el estudiante para que alcance algo" (IN4-H2).

\section{Sobre la competencia mediática}

Las ideas iniciales formuladas por los participantes sobre la competencia mediática fueron: "aquella persona competente mediáticamente es aquella persona que sabe utilizar de manera adecuada y sabe manejar un programa" (IN2-M1) o que el docente debe enseñar "el buen uso y saber qué usar. Por ejemplo, ellos pueden tener al alcance mucha tecnología, [...], pueden saber mucha tecnología, pero no saben guiar muy bien qué programas existen para que puedan facilitar esas capacidades" (IN2-M2).

Identificaron y reconocieron que la abundancia de información puede generar problemas al seleccionarla y diferenciarla: "creo que no todo lo que tenga al alcance lo va a utilizar. Sobre todo, [deben aprender a] seleccionar" (IN3-M5). En el mismo orden de ideas, quedó establecido que parte del proceso era sentirse capaces de discriminar las fuentes de acuerdo con su fiabilidad:

Conocer que no toda la información que se presenta en internet es información que le va a servir al estudiante o que es verídica. En ese caso el estudiante tendría que saber que hay información en internet que no es confiable, entonces, creo que si tiene en la cabeza eso podrá discernir algunas fuentes o medios para buscar (IN1-M5).

Por ello, precisan el rol orientador que deben asumir como docentes, contribuyendo a la reflexión y el análisis:

En la actualidad va a ser muy difícil que los programas chatarras ["televisión basura"] desaparezcan, pero lo que sí se podría hacer es que por medio de la educación se les incentive a los estudiantes a identificar qué cosas están mal y qué cosas están bien, y que rescaten lo positivo de ese programa. De esa manera, estaríamos aportando a que el estudiante pueda desarrollarse mejor (IN2-M8).

A lo largo de la conversación se motivaron y fueron elaborando otros aspectos de la competencia, como "favorecer la capacidad de interpretar [...] Y lo otro sería que, gracias a esa interpretación, otra capacidad podría ser explorar" (IN2-M3]. Asimismo, "la capacidad, relacionada al enfoque sociocrítico, donde el estudiante debe ser un ser autónomo y reflexivo de su propio conocimiento, que no vea que la información que se le da en las aulas es absoluta, sino relativa y dispuesta al cambio" (IN1-M8). 
Una estudiante apuntó que la competencia mediática, en este mismo punto, debería ayudarlos también a enfocar sus prácticas mediáticas:

El [estudiante] chiquito ya tiene cierta capacidad, o cierto desarrollo, de lo que es mediático [...] Por ejemplo, ellos dicen "yo sé usar muy bien el Facebook", pero cómo lo usas, para qué lo usas, cuál es el fin, cuál es la finalidad por la que lo está utilizando, no es algo que le ayude a desenvolverse de una forma que no lastime su integridad (IN2-M2).

Menos frecuentes en la conversación fueron los temas que relacionan la educación mediática con la producción y la expresión. Solamente una participante señaló el vínculo de esta competencia: "con la creatividad, porque nosotras nos comunicamos, también, a través de la creatividad para hacer cualquier trabajo" (IN3-M6); mientras que otra lo hizo con la innovación, entendida como la capacidad de, a partir de un recurso, encontrar

...nuevos modos para aplicarlo, utilizarlo, y eso porque estamos en un periodo de incertidumbre. [...]

Ya no tienes que bombardear con información, como si [el niño] fuera un diccionario viviente, sino de cómo [lo] preparas y lo adaptas para que se enfrente a los diferentes contextos que surgen (IN1-M6).

\section{El autodidactismo como estrategia para formarse en medios}

Fue señalada la necesidad de una actualización permanente: "es necesario una constante capacitación o actualización frente a las modificaciones que se va dando a lo largo del tiempo. Ser autodidacta" (IN3-H1).

Este autodidactismo quizá comprenda implícitamente la dimensión actitudinal y se desprenda del paradigma de aprender a aprender. Como señaló un estudiante, importa cómo "explorar para no limitarse, sino para poder seguir descubriendo cosas" (IN2-M3).

Es palpable el sentimiento de desventaja de los futuros docentes frente al manejo tecnológico de los alumnos, más fluido y versátil. Esto produce tensiones intrínsecas. Unas alumnas declararon que, al aplicar una encuesta en una institución educativa sobre empleo de recursos tecnológicos, los docentes no se sentían capaces de utilizar el aula de innovación. El director comentó: "el aula está, los recursos están y el colegio ha hecho una inversión para tener esos recursos, pero que los docentes tenían miedo de utilizar los recursos, no se sentían con la capacidad suficiente para poder emplearlos" (IN1-M4). Por otro lado, otra intervención indica al respecto: "Creo que de cierta manera tienen miedo porque ahora los estudiantes vienen con el celular, y lo tienen bajo la carpeta" (IN3-M1).

Para muchos, la mayoría de los elementos que podrían configurar una competencia mediática son nuevos o desconocidos, aunque podían intuir de qué tratan. Sin embargo, su percibida "incapacidad" de poner en práctica acciones concretas con tecnologías digitales les resulta frus- 
trante, pues consideran que ni siquiera saben, en muchos casos, cómo manipularlas: “A veces yo quiero un diseño específico, como yo quiero, que lo hago muy bien en papel, pero pasarlo a la computadora me demoro una eternidad" (IN4-M3).

Esto ha promovido, por otro lado, que en la práctica muchos aprendan por sus propios medios:

ahorita yo enseñaré a diseñar páginas web a los chicos y hay un servidor que se llama Apache. Yo no sé mucho de eso, de hace días estoy averiguando (IN4-M5)

Como sabemos, el tiempo avanza, la información cambia y la tecnología también, entonces siempre hay nuevas cosas que aprender. Que [el docente, entonces,] reciba capacitaciones o que dependa de él estar al tanto lo nuevo que hay en el mundo (IN1-M5).

Pero también esta mirada autocrítica sobre sus capacidades es leída como un reto que demanda la profesión docente que han elegido: "Los chicos no van a querer aprender de alguien que ellos van a sentir que no es como ellos" (IN1-M2).

Asimismo, hay espacios para la crítica hacia la idea facilista de las competencias adquiridas de manera natural y espontánea, relacionadas con el nativo digital: "el solo hecho del contacto no le da al niño las capacidades para que el niño sea competente digitalmente" (IN1-M4).

\section{La EM como una deuda en los planes de estudio}

Muchos participantes reconocieron haber aprendido acerca de los medios, aunque en su dimensión tecnológica: "en [la asignatura de] informática tocamos sobre el manejo de ciertos programas. También [...] herramientas para que puedas enseñar didácticamente a los alumnos. Programas como Excel, PowerPoint y Word" (IN2-H1). Añaden:

Mis alumnos saben [usar programas ofimáticos] superficialmente, o quizá a fondo, entonces me gustaría como parte de la educación que se pudiera aprender más, que a nosotros como docentes nos capaciten más en ello. Para poder darle un conocimiento más de lo que ya aportan los estudiantes. Porque quizá yo voy a una clase y enseño tal cosa, y el estudiante ve que yo le enseño y él me enseña. Lo cual no estaría mal. No. Pero mi alumno va a tener dudas y yo voy a tener el deber de solventar esas dudas (IN4-M2).

Más allá de las clásicas asignaturas de informática o TIC, se citaron otros recursos concretos trabajados en alguna clase por iniciativa del profesor de asignatura, pero no como parte del programa oficial. Por ejemplo, la selección de algún vídeo de YouTube para analizar en clase, o el uso de Google Académico para investigar, el programa PowTown para hacer presentaciones, o alguna actividad de robótica básica. En algún caso se mencionó un taller optativo donde "enseñan a hacer historietas, videojuegos educativos para los niños" (IN2-M1). 
De las instituciones educativas con las que trabajamos sólo dos ofrecen asignaturas que podríamos vincular directamente con la EM más allá de la dimensión tecnológica. Una de ellas ofrece la asignatura "Lenguajes de los medios". Dos participantes del grupo focal que la habían cursado explicaron sus contenidos: "vemos mucho lo que es el cine, la radio, la televisión, la función que [cumplen] en la educación" (IN3-M2); también estudian "cómo los medios (cine, televisión, radio) pueden influir en la manera en la que el docente tiene más herramientas para llegar más a sus estudiantes" (IN3-H1). Adicionalmente, esta misma institución organiza un taller de cine abierto a la sociedad Ilamado "ValorArte", donde los participantes analizan películas vinculadas a la espiritualidad (por el origen religioso de ese centro de estudios) y a otro abanico grande de temas sociales. Los estudiantes que participaron en la asignatura o en el taller, los valoraron positivamente porque sienten que cuentan con más recursos para analizar los medios comprendiendo sus sentidos y códigos, y para diseñar experiencias de aprendizaje con sus propios estudiantes.

En casos aislados, los medios de comunicación fueron materia de análisis de otras asignaturas no relacionadas directamente con la EM:

El año pasado llevamos el curso de Ética y tocamos ese tema, de los medios de comunicación y cómo es que la persona debía acceder [a lo] que presentaban los programas. Entonces, veíamos si la culpa era de los programadores que ponen esos programas que no tienen contenido educativo o de la persona que accede. Y decían que la culpa era de las personas porque cada uno está libre de decidir lo que va a ver o no. Entonces, decían otras personas, a nivel del Perú, lo que más tienen los medios son programas basura y poco educativos. Entonces, qué es lo que voy a ver aparte de estos programas... lo único que me toca a elegir es lo que me presentan (IN3-M4).

Al respecto, otros participantes de esa misma conversación señalaron que era muy extraño encontrar compañeros que trabajen en formatos distintos al impreso, aun cuando era algo que buscaba promoverse desde sus propios profesores:

Para el curso de "Estrategias para aprender a aprender" presentaremos un portafolio virtual. Vamos a crear un documento donde vamos a establecer estrategias de aprendizaje para cursos específicos, para niveles específicos y lo vamos a subir a YouTube (IN1-M1).

El ciclo pasado en el curso de Procesos cognitivos teníamos un portafolio [documentos escritos, resumen de lecturas y más]. Nos dieron la opción de presentarlo en virtual o físico. Pero son pocas las personas que prefirieron hacer el portafolio virtual, porque lo físico les facilita mucho más [para dar el examen con material]. [Se remite al dominio del recurso]. No se complican mucho al hacerlo en físico (IN1-M3).

En la misma línea, se consideró una barrera el hecho de que no halla un consenso en el sistema educativo sobre qué medios son más apropiados o pertinentes para cada situación de 
aprendizaje, lo que produce al mismo tiempo una contradicción. A veces les permiten a ellos mismos utilizar el teléfono móvil en clase: "en el curso de 'Currículo' nos pidieron investigar y buscamos información en distintos medios y eso nos permitió hacer un comentario crítico" (IN2M5), pero saben que muchos colegios no tienen una política definida sobre este tema:

Nos dan mucha tecnología, nos enseñan bien la tecnología, pero nos limitan, vamos a un colegio [y nos dicen] "no, no pueden usar celulares, si no te lo decomiso hasta fin de año". Entonces, ¿cómo hago mi clase con los chicos y cómo me cercioro de que el alumno está trabajando lo que le estoy pidiendo y no desviarse del tema? (IN4-M3).

Todos tienen celular, pero la mayoría de los estudiantes piensa que el celular es sólo para mensajear, para usar Facebook, Twitter, pero no le dan una visión educativa al celular. Voy a una clase y en vez de buscar una palabra que no he entendido o seguir lo que el docente está hablando, me distraigo en cosas banales. Entonces, quizá por eso acá en el Perú se dé ese tipo de prohibición porque los estudiantes no tienen claro a qué van al colegio (IN4-H4).

Por otro lado, una estudiante dijo espontáneamente que lo que veníamos discutiendo le recordaba ciertos contenidos televisivos sobre los que ha discutido en alguna clase o con otros compañeros:

Cuando usted [se refiere a los autores de este artículo] llegó al salón y habló de la EM se me vino a la cabeza Combate, esto es guerra [franquicia de programas televisivos del género reality con muy alta sintonía en el Perú] y pensaba en la educación que ellos presentan a la población. Es grosero, es una basura. No responden bien a las preguntas, son hedonistas, dejan de lado el valor de la mujer (IN1-M2).

Antes de ello, algunos compañeros señalaron que también sería relevante discutir, por ejemplo, "cómo influyen en los procesos del niño, en la formación de estereotipos, en procesos cognitivos, [cómo los niños] se vuelven más dependientes electrónicos" (IN1-M1). Otro estudiante retrucó:

Si no se puede con el enemigo, unámonos a ellos... Por ejemplo, a partir de los dibujos que ellos conocen, desarrollar mi clase. Pensaba especializarme en primero y segundo grado. Por ejemplo, Marshall y el oso [un dibujo animado] me facilitaría trabajar el desarrollo emocional en los niños (IN1-M4).

En todo caso quedó demostrado que existe un déficit muy grande en materia de contenidos vinculados con la EM. Como concluyó un participante: "tenemos que autoeducarnos, porque [la EM] tiene que ser transversal, teoría y práctica. Eso... utilizarla en todos los cursos, en todas las ramas, no sólo en las TIC y en los demás cursos lo dejamos de lado" (IN4-H2). Asimismo, 
como señaló otro alumno: "si queremos profundizar en la EM hay algo que es esencial, es ir a la base. Porque si yo quiero enseñar al estudiante bien, tenemos que instruir bien al docente" (IN4-H3).

Como ejercicio proyectivo les propusimos ordenar algunas palabras clave vinculadas con los medios y las tecnologías digitales, para discutir sobre su grado de cercanía con las mismas. Discutimos si habían sido vistas en algunas asignaturas o talleres de la carrera. Las palabras que presentamos las obtuvimos de la revisión de literatura y constituyen conceptos o tópicos sobre los que se viene reflexionando en el ámbito de la educación mediática. El resultado, como se aprecia en la imagen 1, deja ver que muchos de los conceptos que son tendencia en el mundo mediático (como transmedia, big data, gamificación o el internet de las cosas) les resultan poco conocidas o ajenas.

Imagen 1. Palabras clave más y menos conocidas por los estudiantes
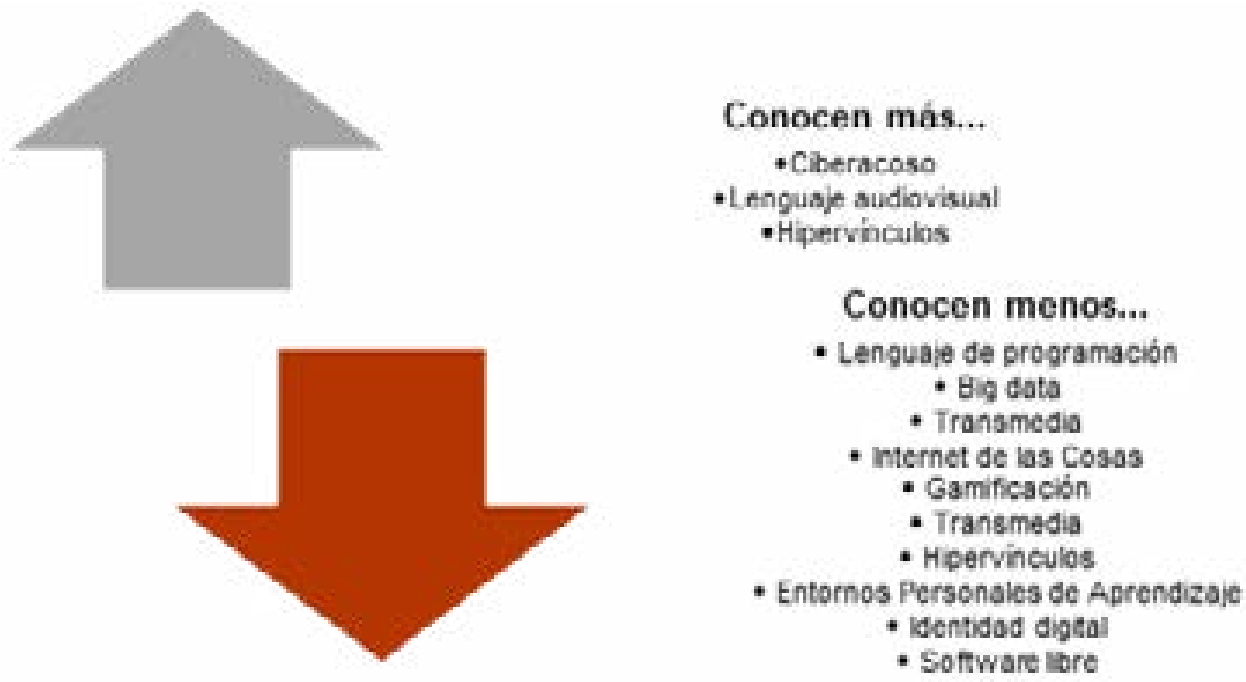

Fuente: Elaboración propia.

\section{Formarse mediáticamente}

Las conversaciones dejaron ver una mirada entusiasta sobre lo que debería ser su formación mediática. Con más intuiciones que certezas sobre sus contenidos, por tratarse de un concepto nuevo para la mayoría, el consenso mayoritario estuvo en la integralidad y la transversalidad de esta preparación.

En cuanto a lo primero, la justificación era la omnipresencia de todos los medios: "están presentes siempre. Entonces, si nosotros nos preparamos a cómo llevar a estos medios, o cómo es un buen uso de estos medios, y cómo podemos implementarlos en las clases... haríamos un mejor rol como docentes" (IN2-M2). 
Sobre la transversalidad, las razones estuvieron más centradas en la concepción de los medios como recursos. A todos les gustaría dominar más programas informáticos y software que les permitan crear material para sus alumnos. En este horizonte, se insistió en el desarrollo de capacidades específicas vinculadas con la producción de contenidos:

Sería bueno un curso dedicado para que los docentes aprendan a hacer sus tutoriales. Para que fácilmente los alumnos tengan acceso si es que no tienen la clase a mano (por equis motivos) ya tienen su tutorial de 5 a 10 minutos en un enlace para la próxima clase (IN3-H1).

Asimismo, como se dijo en el apartado anterior, es vital aprender a leer los medios, no sólo sus mensajes, sino identificar las estructuras de poder subyacentes. En ese sentido, se dijo que "para acceder a cualquier medio de comunicación se tiene que conocer algo de ese programa o de ese medio, qué es lo que nos transmiten en realidad, qué es lo bueno y qué es lo malo" (IN3M2). Para una alumna, el tipo de formación

...debería ser como un curso, una especialización, una manera de hacerles conocer [a los estudiantes] qué hay detrás de esos programas, cómo funciona ese ambiente, y así tú das a entender que no sólo son programas de entretenimiento (los realities), sino que detrás hay una cosa que se mueve (IN3-M4).

Otro elemento, aunque menos recurrente, fue la posibilidad de acceder a una pluralidad de fuentes menos habituales en su dieta mediática. Por ejemplo, "nos deberían enseñar a ver cine independiente porque muchas veces, o sea, sí vemos películas pero las estadounidenses, pero nosotros no sabemos apreciar el cine independiente" (IN2-M5).

Del mismo modo, algunos estudiantes insistieron en el carácter contextual de los medios: "Saber en qué medida están los niños con la tecnología para poder adaptarme a ellos. Pero si me voy a una zona rural, allí implementaría otras cosas más, quizá la radio, que puede ayudar a potencializar la imaginación" (IN3-M2).

Se dijo también que el cambio de actitud significa que no puede mirarse por encima del hombro a los medios. "A mí me gustaría desarrollar competencias de aprender a aprender, pero profundamente" (IN1-M3]. Precisamente, esa es una de las barreras percibidas para impulsar la EM en las escuelas, además del tema de la infraestructura y el equipamiento, sobre todo por las brechas de acceso conocidas en el país, pues "no es que el Estado brinda todo. Entonces va a afectar la parte económica de los padres. Y, en especial, en provincia por la accesibilidad que no se llegan a brindar bien los servicios. Entonces, en ese aspecto el Estado le falta brindar más apoyo" (IN2-M6).

En relación con el tema actitudinal, es frecuente el reclamo por un cambio de paradigma: "Creo que aún tenemos una formación antigua" (IN1-M1). Al respecto, "es ineludible trabajar en 
la falta de apertura de los docentes tanto en su preparación como de las personas que les compete esa función de brindarlo. Creo que más se podría resaltar la disposición para poder integrar a una práctica educativa todos estos conocimientos" (IN1-M3).

Al igual que en el caso de las autoridades, también los padres fueron mencionados como actores prioritarios. Una estudiante dijo que le gustaría saber "cómo o qué medios puedo trabajar en donde los padres puedan entender y estar con sus niños. Me refiero en cómo hacer llegar a los padres. Cómo capacitarlos" (IN3-M4). Hay, en este punto, un trabajo muy grande que hacer para que muchos padres superen los prejuicios sobre el potencial educativo de los medios: "hay casos, por ejemplo, que los padres no quieren que sus hijos vayan a las cabinas o tengan una computadora porque creen que se van netamente a lo malo y no a lo educativo" (IN2-M1). Del mismo modo, sobre todo por la edad de los menores, el acompañamiento de los padres y madres es crucial para la interacción con las tecnologías, sobre todo acompañando y orientando: "No le vas a pedir a un niño de tres años que investigue: 'Busca en Google"' (IN2-M7).

\section{Discusión}

Si bien los docentes son críticos frente a los medios de comunicación sobre asuntos muy generalizados en la opinión pública, relacionados con la ética y el poder político y económico que representan, también manifiestan el valor que tiene la información en la vida cotidiana y pública y, si bien no es la mayoría, hay quienes diferencian cuánto y en qué casos esta información puede convertirse en aprendizaje. Así, coinciden con la premisa propuesta por la UNESCO (2011, 2021) sobre la urgencia de desarrollar la ciudadanía mediática de los estudiantes atendiendo el enfoque de derechos.

En los fututos docentes aparecen algunas ideas como el acceso a la información como un derecho, así como las posibilidades de promover la creatividad con las TIC, dentro y fuera de la escuela. Por eso, en la línea de lo planteado por autores como Sonia Livingstone et al. (2013) o Carlos Scolari (2018), el maestro debe comprender el medio y acercarse a sus prácticas extraescolares para integrarlas de modo más asertivo y estratégico. Esto se adscribe a las dimensiones propuestas por la UNESCO (2021) en el marco de la Alfabetización Mediática e Informacional.

Un tema recurrente es el de los medios como herramientas. En efecto, y esto es común en todo el sistema educativo, la tecnología es vista como un medio y el docente como un mediador. El que conciban los medios como plataformas para operar y enseñar mejor por sí mismas habla de una formación limitada a ese aspecto, al tiempo que confirma un discurso social que subordina al docente a un rol de administrador de información y tecnologías. Sin embargo, hay quienes relacionan a los medios con capacidades para intervenir, crear y compartir, aunque en menor medida. Esto se ha visto con claridad en el marco de esta pandemia global cuando casi la totalidad del servicio educativo se valió de formatos mediáticos para su continuidad a distancia. Al no comprender las posibilidades de cada medio, sus lenguajes, sus formas de participar en 
la vida de los estudiantes, cada docente hizo lo que pudo con lo que tuvo a mano, desaprovechando la oportunidad para explotar nuevos formatos de producción de trabajos, de evaluación o de reflexión sobre los contenidos que vienen consumiendo, particularmente noticias.

Por otro lado, los propios estudiantes de educación aprecian significativamente el valor de un docente calificado y consideran que las competencias mediáticas se acercan no solamente al acceso a la tecnología sino a un uso más pertinente. Precisan que, ante la abundancia informativa, es necesario desarrollar capacidades de análisis e interpretación, que no surgen espontáneamente y que el rol de la escuela y el maestro resultan fundamentales. De alguna forma, acompañar las tendencias autodidactas del aprendizaje que los chicos ya desarrollan. Esto conversa con los retos planteados por Díaz (2019) para la escuela frente a la sociedad de la incertidumbre y que se hizo patente en el contexto singular de educación a distancia que vivimos.

Por otro lado, los maestros se sienten en desventaja frente a los más jóvenes y tienen temores y resistencias. Esto confirma el calado del discurso de los "nativos digitales" y otros mitos afines que consiguen confundir al docente sobre su rol e importancia. Tomar esto en cuenta es vital para desarrollar estrategias de sensibilización y acompañamiento, no sólo desde la formación inicial sino también desde la formación permanente.

Los futuros docentes se reafirman en su interés y entusiasmo por incorporar la educación mediática en su formación como maestros, más allá del aprendizaje de programas y plataformas específicas, que es lo que suele predominar bajo el enfoque de la "competencia digital". Así, se acercan muy positivamente a la necesidad de intervenir, crear y compartir. Del mismo modo, detectan las contradicciones en la enseñanza que se proyectan luego a las aulas. Se habla de tecnologías, y sin embargo se prohíben y no existen políticas claras en las propias instituciones educativas.

Con más intuiciones que certezas, los docentes van formulando ideas frente a la educación mediática, relacionadas con la omnipresencia de los medios, la transversalidad, la posibilidad de producir contenidos en nuevas plataformas, además de saber también interpretar los medios y sus códigos y discriminar con actitud crítica la pluralidad de mensajes. Ya no miran por "encima del hombro" a los medios, se van haciendo conscientes de que hoy en día se aprende de múltiples formas y en múltiples formatos. Identifican una educación que aún es analógica.

En suma, resulta crucial ofrecer una educación mediática a los maestros que se forman para la educación inicial, más aún porque se advierte que expresan intereses muy claros en capacitarse para ofrecer una educación de calidad $y$, además, tienen una actitud propositiva e interesada. Sobre este punto urge llamar la atención sobre la necesidad de abordar desde el inicio de la formación inicial docente las definiciones y sentidos que debe acompañar toda reforma escolar para resultar efectiva. De lo contrario, se profundiza el divorcio de los nuevos discursos con las fórmulas anquilosadas que se desentienden de la cultura mediática y los aprendizajes extraescolares. Como concluyen Cano y Nájar (2019), la educación mediática en la formación 
escolar no ha sido un tema prioritario en el país, por lo que conviene desarrollar un plan que permita la creación de políticas sostenidas que atiendan a las deficiencias de infraestructura, conectividad y gestión pública, con especial atención a la formación inicial docente. Como apuntan Suárez-Guerrero, Rivera y Ribour (2020), "la evidencia nos está mostrando que, pese a las creencias, sigue existiendo inequidad en el acceso y la distribución de las tecnologías digitales. En este sentido, ¿dónde debiese acentuarse lo digital?, ¿otra vez en los dispositivos o en las competencias?".

Queda pendiente, para futuros trabajos, contrastar estos resultados con las perspectivas de los docentes en ejercicio. Asimismo, extender este tipo de acercamientos cualitativos a los propios estudiantes e incluso correlacionar las mejores prácticas en el contexto de educación virtual remota con lo competencia mediática autopercibida en los propios docentes.

\section{Referencias}

Alfamed, I. (2017). Declaración de Juiz de Fora. http://bit.ly/2Drfas7

Aguaded, I.; D. Jaramillo-Dent; Á. Delgado-Ponce (coords.). (2021). Currículum Alfamed de formación de profesores en educación mediática MIL (Media and Information Literacy) en la era pos-Covid-19. Barcelona: Octaedro.

Andrada, P. (2015). Estado de la cuestión de la formación docente en educación en medios. Comunicación y Medios, 32, 83-99. https://doi.org/10.5354/0719-1529.2015.36700

Bulger, M.; P. Davison (2018). The Promises, Challenges and Futures of Media Literacy. Journal of Media Literacy Education, 10(1), 1-21. http://bit.ly/2rzmlfs

Buckingham, D. (2018). Media Literacy Policy in Europe: Where Are We Going? http://bit.ly/2soOB4E

Cano, A.; R. Nájar (2019). Media Education in Peru: A Field Full of Opportunities. En Mateus, J.; P. Andrada; M. Quiroz (eds.). Media Education in Latin America. Londres: Routledge, 137-150.

Díaz, H. (2019). Transformar la educación: una mirada desde la escuela peruana. Lima: Fundación Santillana. http://bit.ly/2qX4QFQ

Frau-Meigs, D.; I. Velez; J. Flores-Michel (2017). Public Policies in Media and Information Literacy in Europe. Nueva York: Routledge.

Gutiérrez, A.; A.Torrego (2018). Educación mediática y su didáctica. Una propuesta para la formación del profesorado en TIC y medios. Revista Interuniversitaria de Formación del Profesorado, 32(1),15-27. https://www.redalyc.org/articulo.oa?id=27454937002

INEI (2019). Estadísticas de las Tecnologías de Información y Comunicación en los Hogares - Abril, Mayo y Junio de 2019 [informe]. http://bit.ly/2Du6f9r

Livingstone, S.; C. Wijnen; T. Papaioannou; C. Costa; M. Grandío (2013). Situating Media Literacy in the Changing Media Environment: Critical Insights from European Research on Audien- 
ces. En Carpentier, N.; K. Schrøder; L. Hallett (eds.). Audience Transformations: Shifting Audience Positions in Late Modernity. Londres: Routledge, 201-227.

Mateus, J.; C. Suárez-Guerrero (2017). La competencia TIC en el nuevo currículo peruano desde la perspectiva de la educación mediática. EDMETIC, 6(2), 129-147. https://doi.org/10.21071/ edmetic.v6i2.6908

Mateus, J.; W. Hernández-Breña; M. Figueras-Maz (2019) Validation of a Self-Perceived Media Competence Instrument for Pre-Service Teachers / Validación de un instrumento de autopercepción de competencia mediática para docentes en formación. Cultura y Educación, 31(2). 436-464. https://doi.org/10.1080/11356405.2019.1597440

Mateus, J.; W. Hernández-Breña (2019). Design, Validation, and Application of a Questionnaire on Media Education for Teachers in Training. Journal of New Approaches in Educational Research, 8(1), 34-41.https://doi.org/10.7821/naer.2019.1.329

Mateus, J.; M. Quiroz (2021). La "Competencia TIC" desde la mirada de docentes de secundaria peruanos: más que habilidades digitales. Revista Peruana de Investigación Educativa, 13(14), 7-23. https://doi.org/10.34236/rpie.v13i14.266

Scolari, C. (2018). Teens, Media and Collaborative Cultures: Exploiting Teens' Transmedia Skills in the classroom. Barcelona: Universitat Pompeu Fabra. http://bit.ly/2swedwN

Suárez-Guerrero, C.; P. Rivera; M. Ribour (2020). Repensar la educación en tiempos de Covid-19. El Diario de la Educación. https://eldiariodelaeducacion.com/2020/07/29/repensar-la-educacion-en-tiempos-de-covid-19/

Suárez-Guerrero, C.; A. Ros-Garrido; J. Lizandra (2021). Aproximación a la competencia digital docente en la formación profesional. RED. Revista de Educación a Distancia, 21(67). http:// dx.doi.org/10.6018/red.431821

Tondeur, J.; J. van Braak; P. Ertmer; A. Ottenbreit-Leftwich (2017). Understanding the Relationship between Teachers' Pedagogical Beliefs and Technology Use in Education: A Systematic Review of Qualitative Evidence. Educational Technology Research and Development, (65), 555-575. https://doi.org/10.1007/s11423-016-9481-2

UNESCO (2011). Media and Information Literacy Curriculum for Teachers. http://bit.ly/2L6zDH8 UNESCO (2019). Marco de competencias de los docentes en materia de TIC. Versión 3.0. París: UNESCO. https://bit.ly/396mlqO

UNESCO (2021). Media and Information Literate Citizens: Think Critically, Click Wisely! https://bit. ly/2SSynOr 